\title{
Experimental and Theoretical Analysis of a Method to Predict Thermal Runaway in Li-ion Cells
}

\author{
Krishna Shah, Divya Chalise, Ankur Jain* \\ Mechanical and Aerospace Engineering Department \\ University of Texas at Arlington, Arlington, TX, USA. \\ * Corresponding Author: email: jaina@uta.edu; \\ 500 W First St, Rm 211, Arlington, TX, USA 76019 \\ $\mathrm{Ph}:+1$ (817) 272-9338; Fax: +1 (817) 2722952
}

\begin{abstract}
Thermal runaway is a well-known safety concern in Li-ion cells. Methods to predict and prevent thermal runaway are critically needed for enhanced safety and performance. While much work has been done on understanding the kinetics of various heat generation processes during thermal runaway, relatively lesser work exists on understanding how heat removal from the cell influences thermal runaway. Through a unified analysis of heat generation and heat removal, this paper derives and experimentally validates a non-dimensional parameter whose value governs whether or not thermal runaway will occur in a Li-ion cell. The parameter comprises contributions from thermal transport within and outside the cell, as well as the temperature dependence of heat generation rate. Experimental data using a 26650 thermal test cell are in good agreement with the model, and demonstrate the dependence of thermal runaway on various thermal transport and heat generation parameters. This parameter is used to predict the thermal design space in which the cell will or will not experience thermal runaway. By combining all thermal processes contributing to thermal runaway in a single parameter, this work contributes
\end{abstract}


towards a unified understanding of thermal runaway, and provides the fundamental basis for design tools for safe, high-performance Li-ion batteries.

Keywords: Lithium Ion Battery, Safety, Thermal Runaway, Battery Cooling, Thermal Modeling.

\section{Introduction}

Thermal runaway in Li-ion cells is a widely researched phenomenon that presents severe safety challenges [1-3] and often requires conservative design and run-time control of energy conversion and storage devices, resulting in reduced performance [4,5]. Fundamentally, thermal runaway in a Li-ion cell is a cascade of successive processes and reactions that feed into one another through heat generation that increases with temperature, eventually leading to explosion and fire $[1,3]$. Pertinent processes in thermal runaway include decomposition of the solidelectrolyte interface [6], various chemical reactions involving the electrolyte and electrode binder [7-9], and eventually, decomposition of electrolyte [10] and positive electrode active material [11]. A large amount of literature is available on understanding each of these processes $[1,3,6,7,12-13]$. In particular, the reaction kinetics and heat generation profiles of these processes have been widely studied, both theoretically [12] and through experimental measurements using tools such as Differential Scanning Calorimetry (DSC) and Accelerated Rate Calorimetry (ARC) $[6,7,13]$. These processes are modeled using Arrhenius reaction kinetics, with a reaction rate, and hence heat generation rate, that increases exponentially with temperature $[1,3]$. Numerical values of these reaction rates and heat generation rates, as well as their temperature dependence have been determined [3]. Some work also exists on overall heat generation rate measurement from a Li-ion cell at large discharge rates where thermal runaway may be a concern $[14,15]$. 
Some research has also been carried out on thermal modeling and measurements for $\mathrm{Li}$ ion cells in thermal runaway situations $[12,16-21]$. Analytical methods and simulation tools [12,16-18] have been used for modeling thermal behavior of a cell at elevated temperatures. Experiments have been carried out to measure temperature of a cell or an appropriate thermal surrogate [19-21] during runaway. However, not much work has been carried out on connecting heat removal and heat generation processes that occur during thermal runaway. While heat generation is a function of the chemical reactions occurring within the cell, heat removal from the cell comprises of two processes that occur in series $[22,23]$ - thermal conduction within the cell to its outside surface, and heat removal, usually through convection from the outside surface to the ambient. The nature of interaction between these heat generation and heat transfer processes eventually determines the thermal state of the cell, and whether thermal runaway occurs or not. It is critical to model both in a holistic fashion to better understand thermal runaway, and design means to prevent thermal runaway. Specifically, it is of interest to determine what thermal transport properties of the cell and its ambient influence and govern the occurrence of thermal runaway. Such experimentally-verified theoretical limits on the occurrence or avoidance of thermal runaway may result in valuable design tools for safety of Liion cells.

The interaction between heat generation and heat removal has been represented in past papers in the form of a Semenov plot $[24,25]$ that compares the rates of heat generation and heat removal as functions of the temperature of the cell. A Semenov plot uses the imbalance between the two processes - which increase linearly and exponentially respectively as functions of temperature - as the basis for predicting thermal runaway [24,25]. However, this approach assumes the cell to be a lumped thermal mass with uniform temperature throughout the cell 
volume. As shown by recent models [24,25] and measurements [26,27], this may not be an accurate assumption. As a consequence, the Semenov analysis predicts the thermal runaway process to be independent of the thermal conductivity of the cell, which is not accurate in several cases. For example, the Biot number [28] for a typical 26650 cell in natural convection conditions can be estimated to be 2-6.5 based on recent thermal conductivity measurements of the cell $[29,30]$. A value of $B i>1$ indicates strong temperature gradients within the cell, making the lumped mass based Semenov analysis inaccurate. Because the Semenov approach neglects heat transfer within the cell, it has not been possible so far to predict what internal and external thermal conditions are needed to prevent thermal runaway for a specific cell chemistry with wellknown chemical kinetics and heat generation rates as functions of temperature. Accounting for this phenomenon will help optimize materials design from a thermal perspective, and help maintain a balance between internal and external heat transfer towards avoidance of thermal runaway.

This paper carries out theoretical and experimental analysis of thermal runaway in Li-ion cells by extending the Semenov analysis to account for heat transfer within the cell. The governing energy conservation equation that accounts for both heat generation and heat removal processes is solved to determine a non-dimensional parameter - which is named the Thermal Runaway Number $(T R N)$ - whose value is shown to govern whether thermal runaway will occur or not. This parameter includes contributions from heat transfer processes within and outside the cell, as well as the rate of increase in heat generation with temperature. Results indicate that thermal conductivity within the cell is a critical thermal property governing runaway. Experiments that implement a temperature-dependent heat generation with a controllable temperature slope are carried out to validate the theoretical model. These experimental data are 
in good agreement with theoretical results, and demonstrate successful avoidance of thermal runaway through changes in the convective heat transfer coefficient external to the cell. Design guidelines that predict the parameter space in which thermal runaway is prevented are developed using the model. The experimentally-verified fundamental insights in this paper may lead to design guidelines for thermal properties of the cell and its ambient for prevention of thermal runaway.

\section{Mathematical Modeling}

Consider a cylindrical Li-ion cell of radius $R$, radial thermal conductivity $k_{r}$, heat capacity $C_{p}$ and mass density $\rho$. The cell experiences a temperature-dependent internal heat generation rate $Q(T)$, and is being cooled at the outside surface with a convective heat transfer $h$ due to a mechanism such as coolant flow. The interest is in determining the parameter space within which the cell will not undergo thermal runaway, i.e. the cell temperature does not become unbounded. In this case, the governing energy equation for the temperature rise $T(r, t)$ in the cell is given by

$$
k_{r}\left(\frac{\partial^{2} T}{\partial r^{2}}+\frac{1}{r} \frac{\partial T}{\partial r}\right)+Q(T)=\rho \cdot C_{p} \frac{\partial T}{\partial t}
$$

subject to

$$
\frac{\partial T}{\partial r}=0 \quad \text { at } r=0
$$

and

$$
k_{r}\left(\frac{\partial T}{\partial r}\right)=h \cdot T \quad \text { at } r=R
$$


Equations (1)-(3) can be solved to determine if there is a set of conditions that will prevent thermal runaway by ensuring a bounded solution for $T$ at all times. To do so, a Taylor series expansion of $Q(T)$ is first carried out about a temperature $T=T_{0}$, and second order and higher terms are neglected. This results in

$$
k_{r}\left(\frac{\partial^{2} T}{\partial r^{2}}+\frac{1}{r} \frac{\partial T}{\partial r}\right)+Q\left(T_{0}\right)+\beta\left(T-T_{0}\right)=\rho C_{p} \frac{\partial T}{\partial t}
$$

where $\beta=\frac{d Q}{d T}$ is the slope of $Q(T)$.

In order to solve equation (4), it is noted that the heat generation term can be split linearly into two components, $\left(Q\left(T_{0}\right)-\beta T_{0}\right)$ and $\beta T$. The first component is a constant quantity, which from thermal conduction theory [31] is known to result in a steady state with a bounded temperature field. However, the second heat generation component $\beta T$ increases with temperature, and may lead to an unbounded temperature. Solving only for $T_{2}(r, t)$, which represents the temperature rise due to the second component of heat generation, it can be shown using the technique of separation of variables [31] that

$$
T_{2}(r, t)=\sum_{n=1}^{\infty} C_{n} J_{0}\left(\frac{\mu_{n} r}{R}\right) \cdot \exp \left(\frac{k_{r}}{\rho \cdot C_{p}}\left(\frac{\beta}{k_{r}}-\frac{\mu_{n}^{2}}{R^{2}}\right) \cdot t\right)
$$

where $J_{0}$ is the Bessel function of the first kind of order $0, C_{n}$ are constant coefficients, and $\mu_{n}$ are non-dimensional eigenvalues given by the roots of the equation

$$
B i \cdot J_{0}(x)-x J_{1}(x)=0
$$

where $B i=\frac{h R}{k_{r}}$ is the Biot number [28]. Note that $C_{n}$ in equation (5) are obtained using orthogonality and the initial condition of the temperature field. 
The temperature solution in equation (5) may be either bounded or unbounded depending on the sign of the term within the exponential function in equation (5). This term remains bounded if

$$
\frac{\beta}{k_{r}}-\frac{\mu_{n}^{2}}{R^{2}}<0
$$

for each $n=1,2,3$.

Since the values of $\mu_{n}$ increase with $n$, it is sufficient to require that the first eigenvalue $\mu_{1}$ satisfies equation (7). This condition can be written in terms of a single non-dimensional parameter as

$$
T R N \equiv \frac{\beta \cdot R^{2}}{k_{r} \mu_{1}^{2}}<1
$$

Thus, a fundamental, non-dimensional parameter has been derived, which we denote as the Thermal Runaway Number $(T R N)$, whose value must be less than one in order to prevent thermal runaway. Since this derivation is based on Taylor series expansion of $Q(T)$ at a specific temperature $T_{0}$, therefore equation (8) must be satisfied over the entire temperature range of operation to ensure that thermal runaway does not occur throughout.

The non-dimensional parameter $T R N$ in equation (8) is a combination of properties of thermal transport within the cell $\left(k_{r}\right)$, thermal transport from the cell surface to the outside $\left(\mu_{1}\right)$ and the kinetics of heat generation $(\beta)$, as well as the cell geometry $(R)$. These parameters combined in the manner shown in equation (8) determine whether thermal runaway occurs or not. Li-ion cell design and run-time thermal management must be carried out to either reduce the numerator or increase the denominator or both in equation (8). 
The first eigenvalue $\mu_{l}$ can be determined by solving the transcendental equation (6) once Biot number, comprising the cell radius, $R$, thermal conductivity, $k$, and outside convective heat transfer coefficient, $h$, is known. $\mu_{l}$ is plotted as a function of the Biot number in Supplementary Figure 1. As $B i$ increases, $\mu_{l}$ increases, but eventually saturates. This means that regardless of how strong convective heat transfer outside the cell is, the value of $\mu_{l}$ will at most be 2.403 . Simply continuing to increase $B i$ does not necessarily improve the chances of preventing thermal runaway.

Note that equation (8) is derived by assuming the Li-ion cell to be an infinite cylinder, which for most cell geometries such as 18650 and 26650 cells is a reasonable assumption. If a cylinder of finite height $H$ is modeled instead, equation (8) must include additional contributions from the axial eigenvalue as follows:

$$
\frac{\beta R^{2}}{k_{r}\left(\mu_{1}^{2}+\lambda_{1}^{2}\left(\frac{R}{H}\right)^{2} \gamma^{2}\right)}<1
$$

where $\lambda_{I}$ is the first axial eigenvalue, determined based on convective conditions on the top and bottom surfaces, $\gamma$ is the thermal conductivity ratio $k_{z} / k_{r}$, and $H / R$ is the aspect ratio. As expected, for large aspect ratio, equation (9) reduces to the infinite cylinder result given by equation (8).

A theoretical derivation in Supplementary Information shows that if $B i<<1$, i.e., thermal conduction within the cell is neglected and the entire cell is treated as a lumped thermal mass, as has been done in Semenov plots in previous papers [24,25], equation (8) reduces to the following simpler form:

$$
\beta V<h \cdot A
$$


which corresponds to the Semenov analysis presented in the past that treats the body as a lumped thermal mass. Equation (10) states simply that to prevent thermal runaway, the rate of heat generation must be lower than the rate of heat removal. However, this simplified condition is accurate only for a lumped thermal mass because it does not account for thermal transport within the cell. By accounting for this, the model presented here generalizes the Semenov analysis, and offers a more realistic and accurate prediction of thermal behavior of a Li-ion cell.

The next section discusses experiments carried out on a 26650 thermal test cell to experimentally validate the model presented in this section. Results and discussion are presented in Section 4.

\section{Experiments}

Experimental investigation of the influence of $T R N$ on thermal runaway presents several challenges. It is difficult to accurately control and measure heat generation rate in a Li-ion cell, particularly one that changes with temperature, as required for such experiments. Further, measurement of temperature inside the cell is not straightforward [14,27]. As a result, these experiments utilize a thermal test cell that mimics heat generation in a $\mathrm{Li}$-ion cell and provides the capability of close control of temperature-dependent heat generation rate.

\subsection{Fabrication of Thermal Test Cell}

A thermal test cell is designed and built to closely mimic the thermal behavior of a 26650 Li-ion cell. The thermal test cell has similar geometry and thermal transport properties as a $26650 \mathrm{Li}$-ion cell. Heat generation in the thermal test cell occurs due to Joule heating in a wound resistive metal sheet, which makes it possible to measure and change the heat generation rate as a function of temperature by varying the heating current. A thin foil of 304 Stainless Steel and thickness $0.025 \mathrm{~mm}$ is first insulated by adhering Kapton tape on its surface. The foil-Kapton 
sandwich is rolled around a thin rod to form a cylinder of height $65 \mathrm{~mm}$ and radius $13 \mathrm{~mm}$. Seven T-type thermocouples are placed during the rolling process at different radii. Thin metal wires are soldered to the two ends of the foil to provide electrical access to the metal foil. The roll is then lowered inside an Aluminum casing of the same dimensions as a $26650 \mathrm{Li}$-ion cell, and the thin rod is carefully taken out. The heater and thermocouple wires are routed out of the casing. The remaining volume inside the casing is filled with uncured poly-dimethylsiloxane (PDMS), a commonly used electrically insulating soft polymer, followed by curing for 2 hours at $60{ }^{\circ} \mathrm{C}$. PDMS filling is carried out in two steps in order to expel all air inside the test cell. Once filled with PDMS and cured, the test cell is sealed with an epoxy.

Measurement of thermal conductivity of the thermal test cell is measured to be 0.20 $\mathrm{W} / \mathrm{mK}$ using an adiabatic radial heating method [29]. This value is close to that of a $26650 \mathrm{Li}-$ ion cell $[29,30]$.

\subsection{Temperature-Dependent Heat Generation in Thermal Test Cell}

Electrochemical processes in a $\mathrm{Li}$-ion cell generate heat at a rate that typically increases with temperature due to Arrhenius-type nature of these processes $[1,3]$. In order to obtain a similar temperature dependence of heat generation in the thermal test cell, the heating current, supplied by a LabView-controlled Keithley 2401 sourcemeter is modulated based on temperature measurement from the embedded thermocouples. Figure 1 shows a picture of the experimental setup, and a schematic of information flow between various instruments for control of the heatgeneration rate. The thermocouple is read every one second, and the heating current is changed in order to maintain the heat generation rate according to any desired function of temperature. Simultaneous measurement of heat generation rate is carried out through measurement of input current and induced potential difference. Supplementary Figure 2 shows that through this 
(a)

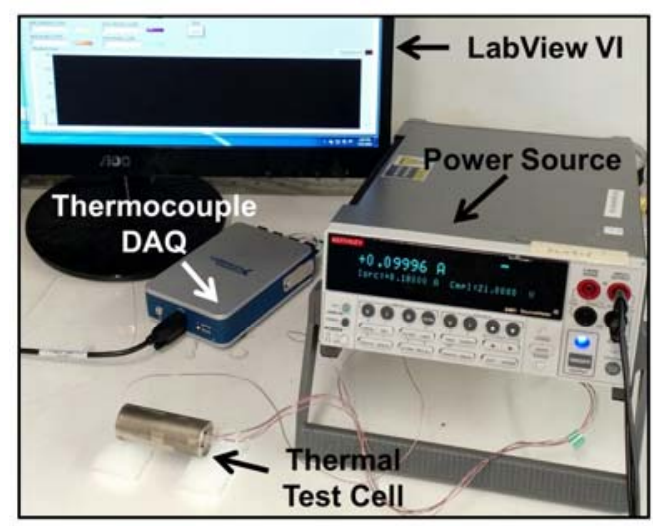

(b)

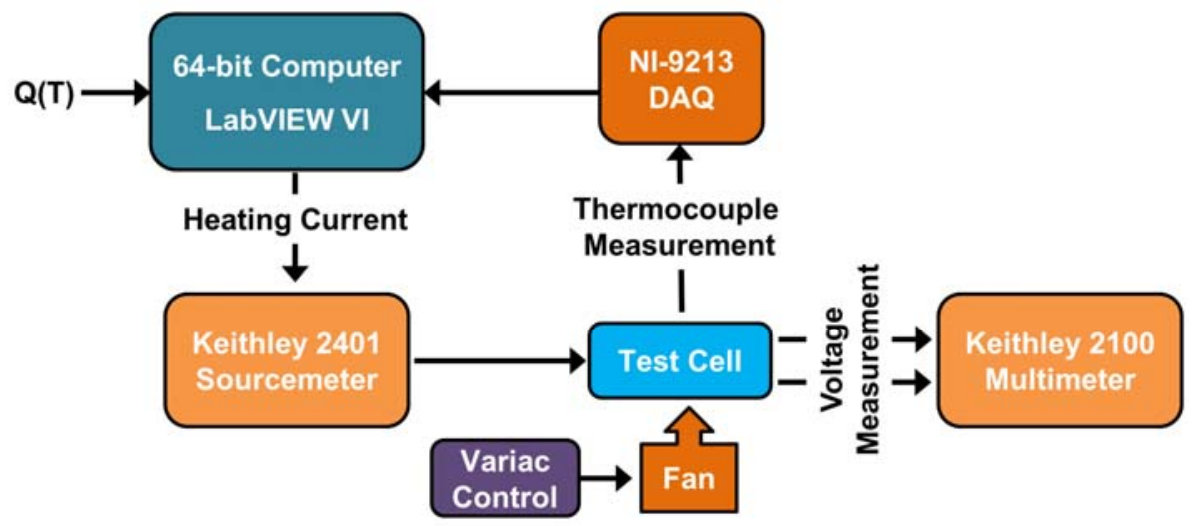

Figure 1. (a) Picture, and (b) Schematic of the experimental setup.

feedback, the heat generation rate in the test cell remains close to the desired profile throughout the experiment, thereby demonstrating the capability of inducing a desired, temperaturedependent heat generation rate in the thermal test cell. Two specific temperature profiles investigated in this work include linearly and exponentially increasing heat generation as functions of temperature.

\section{Results and discussion}

Supplementary Figure 3 compares the temperature rise as a function of time predicted by the analytical model in Section 2 with finite-element model simulations, carried out in ANSYSCFX. This comparison is carried out for linearly increasing heat generation rate at two different values of $\beta$, one which is expected to induce thermal runaway, and one which is not. In both cases, there is good agreement between the analytical model and finite-element simulations.

Figure 2 plots results from temperature measurements on the thermal test cell subjected to linearly increasing $Q(T)$, with different values of the slope $\beta$. Equation (8) predicts that as $\beta$ increases, the value of $T R N$ will increase, and eventually exceed the threshold value of 1 , beyond 
which thermal runaway is expected to occur. This effect is clearly seen in Figure 2 where the value of TRN corresponding to various experiments is shown alongside each curve. For low $T R N$, the cell temperature remains bounded. As $T R N$ increases, the temperature starts to increase due to increased heat generation, but still stays bounded. Once $T R N$ exceeds 1 , however, thermal runaway occurs, with the shape of the temperature curve changing from concave to convex. Experimental data in Figure 2 demonstrate that runaway occurs beyond a threshold value of 1, as expected from the model. In addition, Figure 2 also shows that in each experiment, there is good agreement between experimental data and analytical model over the entire time period.

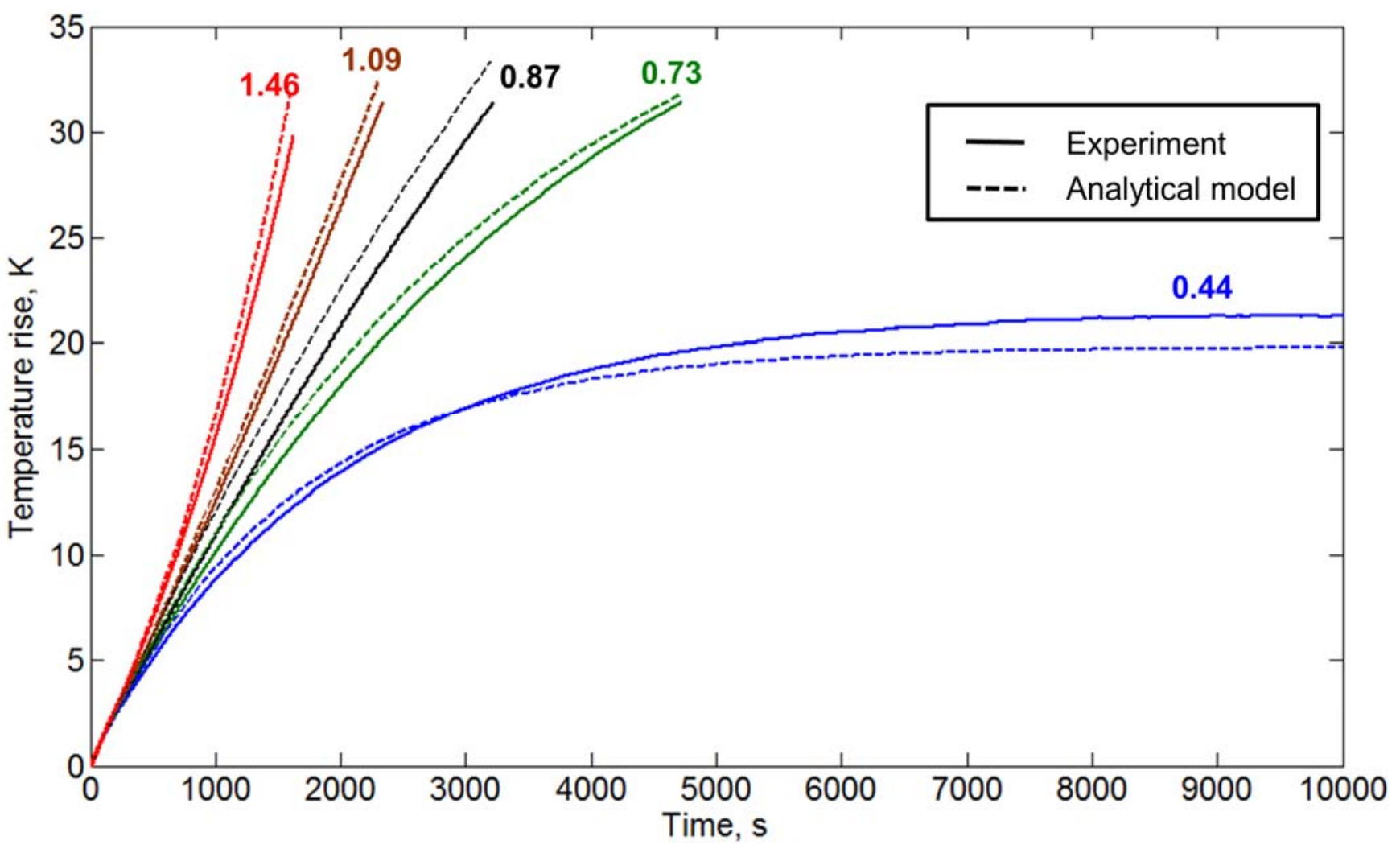

Figure 2. Core temperature of the thermal test cell measured as a function of time for a number of values of $\beta$, ranging from $805 \mathrm{~W} / \mathrm{m}^{3} \mathrm{~K}$ for the left-most (blue) curve to $2685 \mathrm{~W} / \mathrm{m}^{3} \mathrm{~K}$ for the rightmost (red) curve. These data show that thermal runaway occurs when $T R N>1$, and is prevented when $T R N<1$. The value of $T R N$ is shown for each curve. Prediction of temperature profile from the analytical model in Section 2 is also shown for comparison. 
As described in section 2, TRN includes contributions from heat generation rate $(\beta)$, thermal conduction within the cell $(k)$ as well as convective heat transfer on the outside $(h)$. To investigate this further, experiments are carried out at fixed $\beta$ and $k$, but with different cooling conditions on the outside surface of the cell to vary $h$. This is accomplished by providing a cooling fan to blow air over the fan and varying the cooling air speed. Figure 3 plots the temperature of the cell as a function of time for these experiments. As the air speed increases, so does $h$, resulting in reduction in the value of $T R N$ according to equation (8). Eventually, as TRN keeps reducing, as predicted by the model, the cell does not enter into thermal runaway, shown by the bottom three curves in Figure 3. These data further validate the theoretical prediction of a threshold value of $T R N$ to induce thermal runaway in the cell.

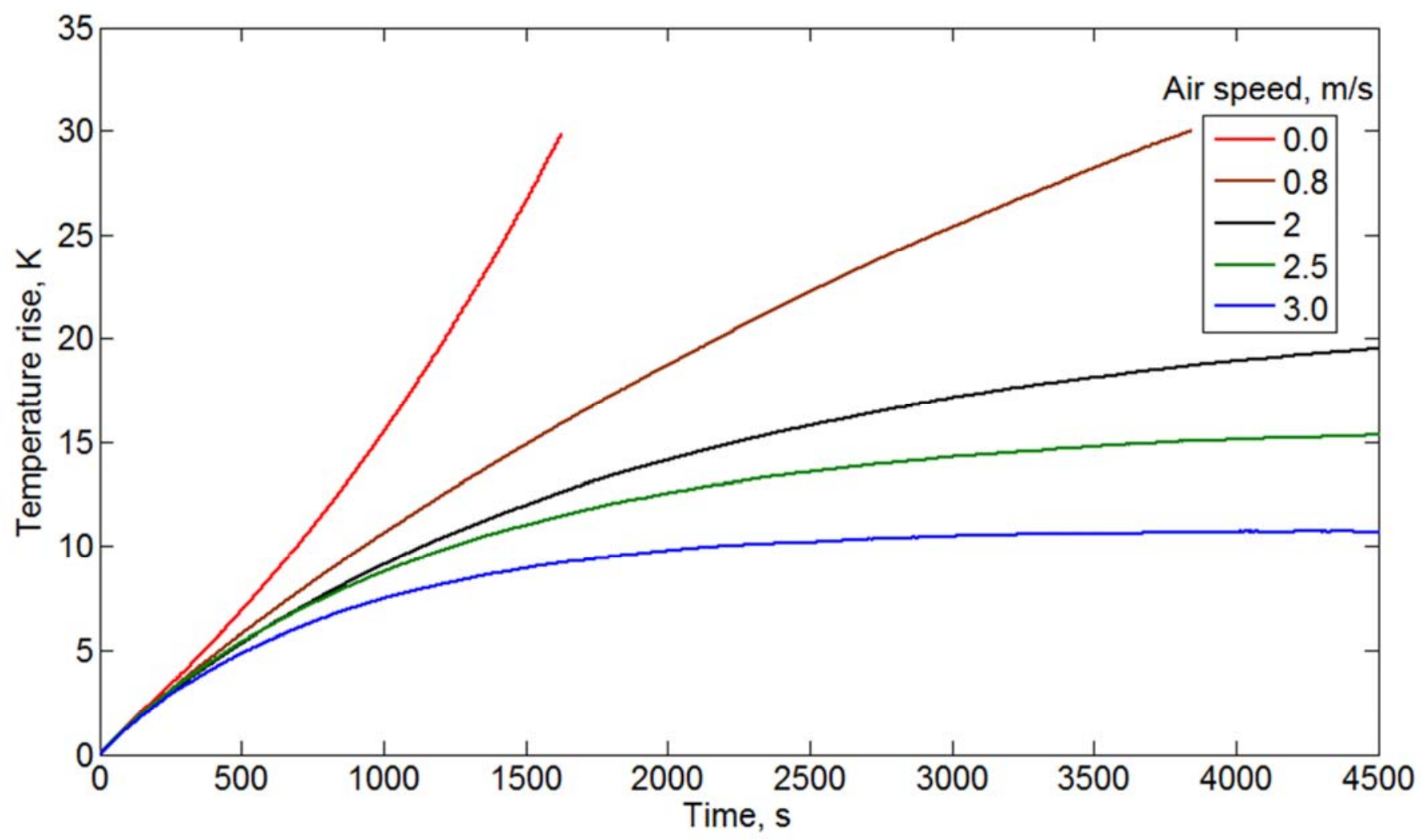

Figure 3. Core temperature of the thermal test cell measured as a function of time for fixed $\beta$ and different convective heat transfer conditions. 
A set of experiments are then carried out using an Arrhenius-type temperature dependence of the heat generation rate, which is more representative of physiochemical processes in a Li-ion cell that are responsible for thermal runaway. In general, the Arrhenius relationship for heat generation rate in a process is given by [32]

$$
Q(T)=Q_{0} \exp \left(-\frac{E_{a}}{R T}\right)
$$

Where $Q_{0}$ is the pre-exponential constant, $R$ is the ideal gas constant, and $E_{a}$ is the activation energy. Note that due to the exponential relationship, $\beta$, the slope of $Q$ is not constant, but increases with increasing temperature.

Two experiments are carried out at two different values of $E_{a}$. The values of $E_{a}$ are chosen such that the resulting $T R N$ corresponding to one of the $E_{a}$ values crosses over the threshold within a specific temperature window, but not for the other. Figure 4(a) plots the measured temperature response as a function of time for both cases. As temperature increases, the heat generation rate increases exponentially, which results in a dynamic value of TRN. The variation in $T R N$ as a function of time is also plotted in Figure 4(a), which shows that for the higher $E_{a}$ case, TRN always stays under 1, and as a result, the measured temperature remains bounded. For the lower $E_{a}$ case, $T R N$ starts at a low value, but increases rapidly, and exceeds the threshold of 1 beyond around 1000s. During the time that $T R N$ remains under 1 , the temperature profile for this case also appears to remain bounded, but, as expected from the theoretical model, once $T R N$ exceeds 1, the temperature distribution becomes unbounded, resulting in thermal runaway. There is an inflexion in the temperature curve at the time that $T R N$ crosses the threshold value of 1. Figure 4(b) investigates this further by plotting the temperature profile for the lower $E_{a}$ value at which thermal runaway occurred in the previous experiment, but with two 

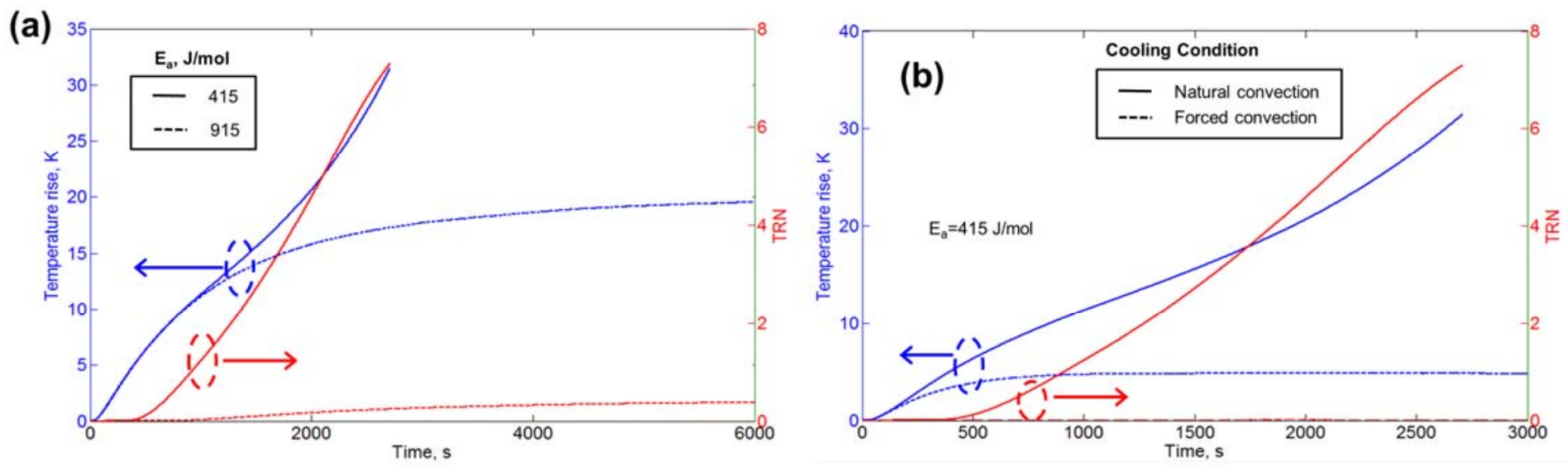

REVISED Figure 4. Experimental measurement of thermal test cell core temperature for (a) two different values of activation energy, $E_{a}$, (b) two different values of convective heat transfer coefficient. Plots also indicate the evolution of TRN with time, and show that thermal runaway occurs when TRN exceeds 1.

different cooling conditions - natural convection and forced convection using a fan. Figure 4(b) shows that thermal runaway that occurred in the previous experiment could be prevented through external cooling, which increases the value of $h$, and hence reduces the value of $T R N$. Figure 4(b) shows that when externally cooled, the value of $T R N$ always stays below 1 , thereby preventing thermal runaway as predicted by the model presented in section 2 .

For a Li-ion cell of a given chemistry and operating at a certain C-rate, there are two thermal transport parameters that influence the rate of heat removal from the cell - the thermal conductivity $k$, which governs the thermal conduction process within the cell, and convective heat transfer coefficient $h$, which governs heat removal from the cell surface to a coolant through thermal convection. It is important to understand how the value of $T R N$ depends on these two parameters. Figure 5 shows a colorplot of $T R N$ in the $h-k$ space relevant for most practical cooling strategies for $\beta=6000 \mathrm{~W} / \mathrm{m}^{3} \mathrm{~K}$. A curve corresponding to $T R N=1$, which is the threshold that separates the safe region (to the top and right of the $T R N=1$ curve) and unsafe region (to the 


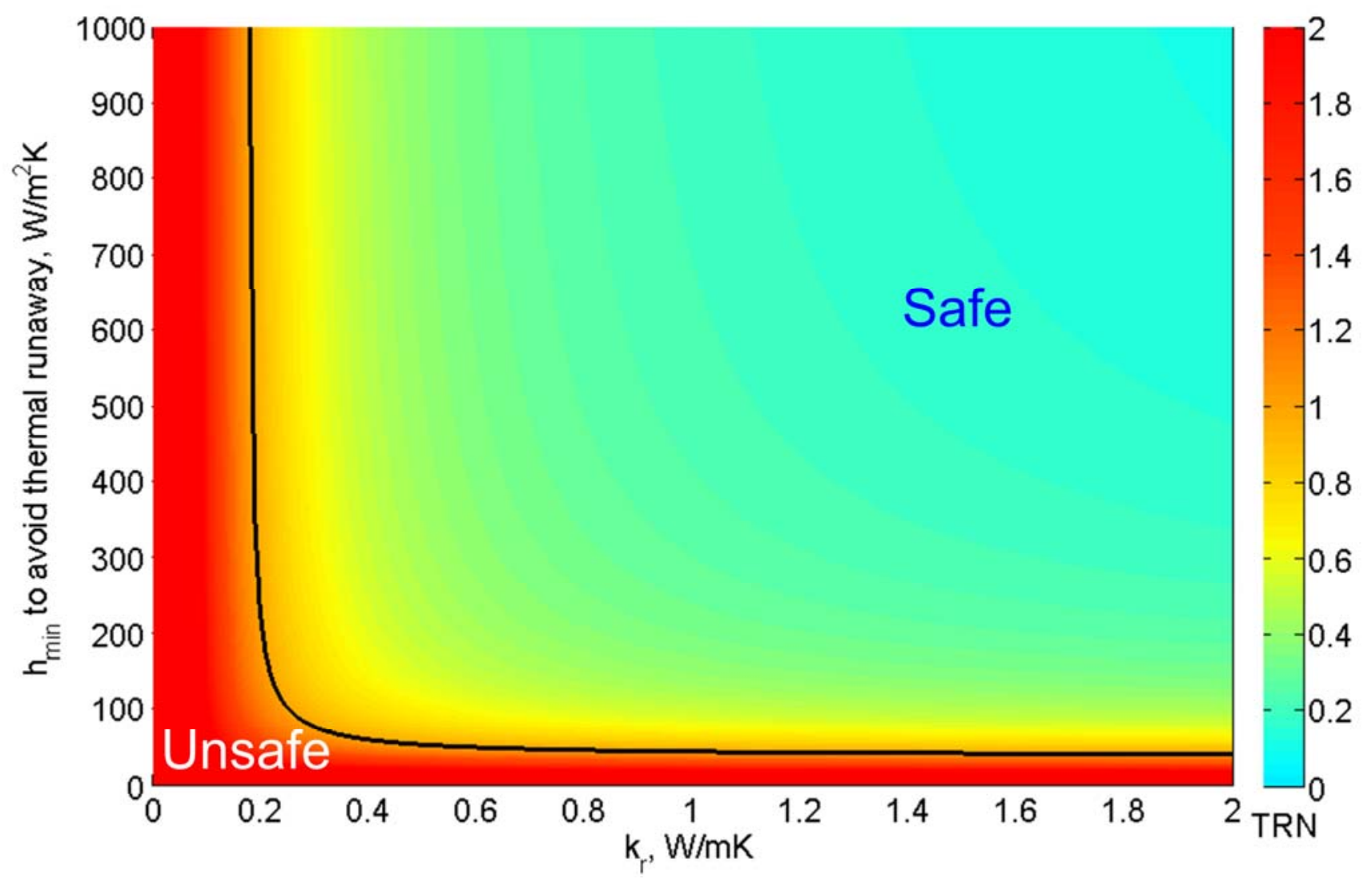

REVISED Figure 5. Colorplot of $T R N$ in the h-k space. The $T R N=1$ curve that separates safe and unsafe regions is also shown.

bottom and left of the curve) of this space is also shown. This curve provides a useful design guideline for thermal management of Li-ion cells by specifying what values of thermal transport parameters are expected to be sufficient to prevent thermal runaway at a given value of $\beta$. Further, in case thermal runaway is to be expected, this figure shows what parameters need to be changed and by how much in order to cross into the safe region of the $h-k$ space shown in Figure 5. In general, the $\mathrm{TRN}=1$ curve that separates the safe and unsafe regions shifts towards the top and right as $\beta$ increases, leaving lesser and lesser of the $h-k$ space in the safe region. For reference, recent measurements have reported the radial thermal conductivity of 26650 cells to be in the range of $0.2-0.65 \mathrm{~W} / \mathrm{mK}[29,30]$. On the $h$ axis, natural convection cooling results in a value of $h$ in the range of $10-100 \mathrm{~W} / \mathrm{m}^{2} \mathrm{~K}$ [28], while forced convection values for $h$ are larger, depending on the nature of coolant fluid and flow speed [28]. 

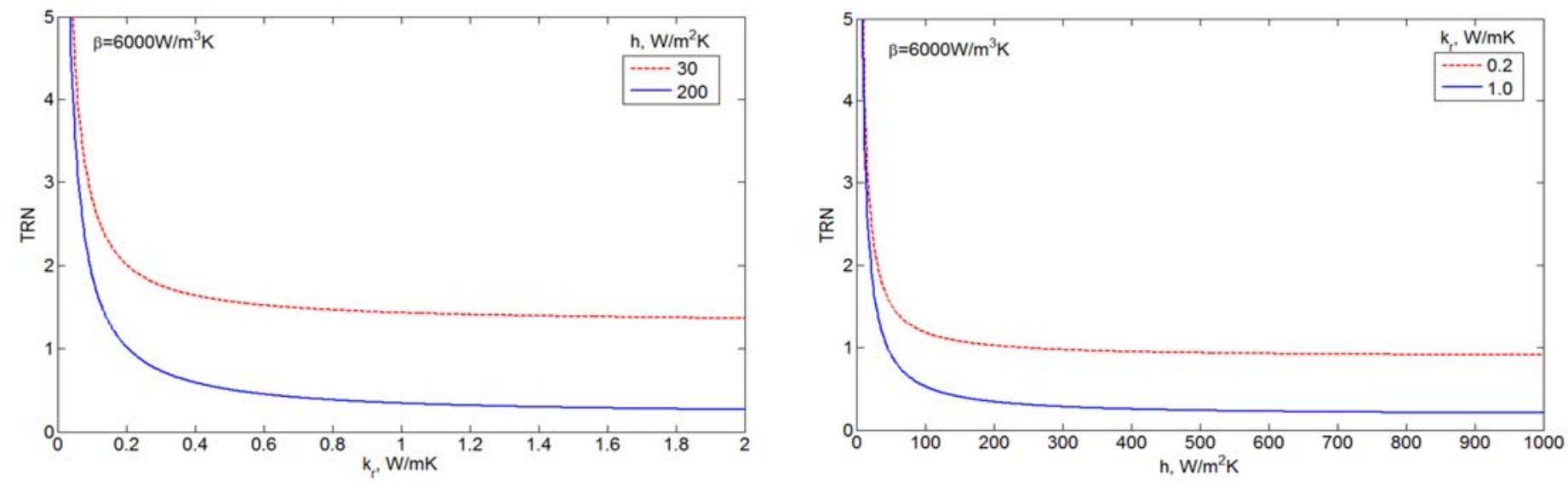

REVISED Figure 6. (a) $T R N$ as a function of cell thermal conductivity, $k$ for two different values of the convective heat transfer coefficient, $h$; (b) TRN as a function of the convective heat transfer coefficient, $h$ for two different values of the cell thermal conductivity, $k$.

Figures 6(a) and (b) plot TRN as a function of $h$ and $k$ respectively while the other parameter is fixed. Both figures use $\beta=6000 \mathrm{~W} / \mathrm{m}^{3} \mathrm{~K}$. Figure 6(a) shows that at a fixed $k=0.2$ $\mathrm{W} / \mathrm{mK}, T R N$ reduces as $h$ increases, i.e., as the cell is cooled more and more aggressively, and dips below the threshold value of 1 at around $h=233 \mathrm{~W} / \mathrm{m}^{2} \mathrm{~K}$. This transition can be made to occur at a lower $h$, if the thermal conductivity of the cell could be improved. At $k=1 \mathrm{~W} / \mathrm{mK}$, Figure 6(a) shows that a heat transfer coefficient of $45 \mathrm{~W} / \mathrm{m}^{2} \mathrm{~K}$ is sufficient to prevent thermal runaway. Similarly, Figure 6(b) shows that increasing the cell thermal conductivity reduces $T R N$ when $h$ is held constant. Both Figures 6(a) and 6(b) exhibit a saturation effect in $T R N$ as either $h$ or $k$ continue to increase. $T R N$ for a cell with too low thermal conductivity might not dip below the threshold value of 1 at all regardless of how effectively it is cooled on the outside. Note that increasing $h$ requires improved heat transfer external to the cell, whereas increasing $k$ requires improving material and interfacial thermal resistances within the cell [33]. 
The interplay between $h$ and $k$ for reducing TRN and preventing thermal runaway is further illustrated in Supplementary Figure 1, which plots the first root $\mu_{l}$ of equation (6) as a function of the Biot number, $B i$. As $B i$ increases, there is a sharp increase in $\mu_{l}$, resulting in significant benefit to $T R N$, but beyond a value of around 30, further increase in $B i$ does not significantly increase $\mu_{1}$. This illustrates the inherent limitations in using convective cooling to improve thermal runaway performance of the cell, for which $k$ is a constant. Once the heat transfer coefficient is somewhat large, its role in preventing thermal runaway saturates, and further benefit must come from thermal-friendly cell design, for example by improving thermal transport through materials and interfaces inside the cell.

When carrying out an effective thermal design of the cell to prevent thermal runaway, parameters such as cell radius and thermal conductivity of the cell are usually fixed, whereas the convective heat transfer coefficient can be increased somewhat, for example, by provided additional coolant flow on the outside of the cell. In such a case, it is important to determine $h_{\text {min }}$, the minimum value of the convective heat transfer coefficient that can sustain a given heat generation rate without causing thermal runaway. Figure 7 plots $h_{\min }$ as a function of $\beta$ for a number of values of the cell thermal conductivity, $k$. This plot shows that for a given value of $k$, as $\beta$ increases, the minimum value of $h$ needed to prevent thermal runaway increases slowly at first, but then very sharply, eventually becoming too large to be practicable. Since mul has a theoretical maximum value of 2.405 (see Suppl. Fig 3), therefore, for a given $R$ and $k$, the maximum $\beta$ that a cell can sustain without thermal runaway is given by $\beta_{\max }=5.78 \cdot \mathrm{k} / R^{2}$, which requires very strong convective cooling corresponding to a Biot number of around 50 or more. If the expected $\beta$ of a cell is greater than $\beta_{\max }$, then, even the best possible external cooling may not be effective. 


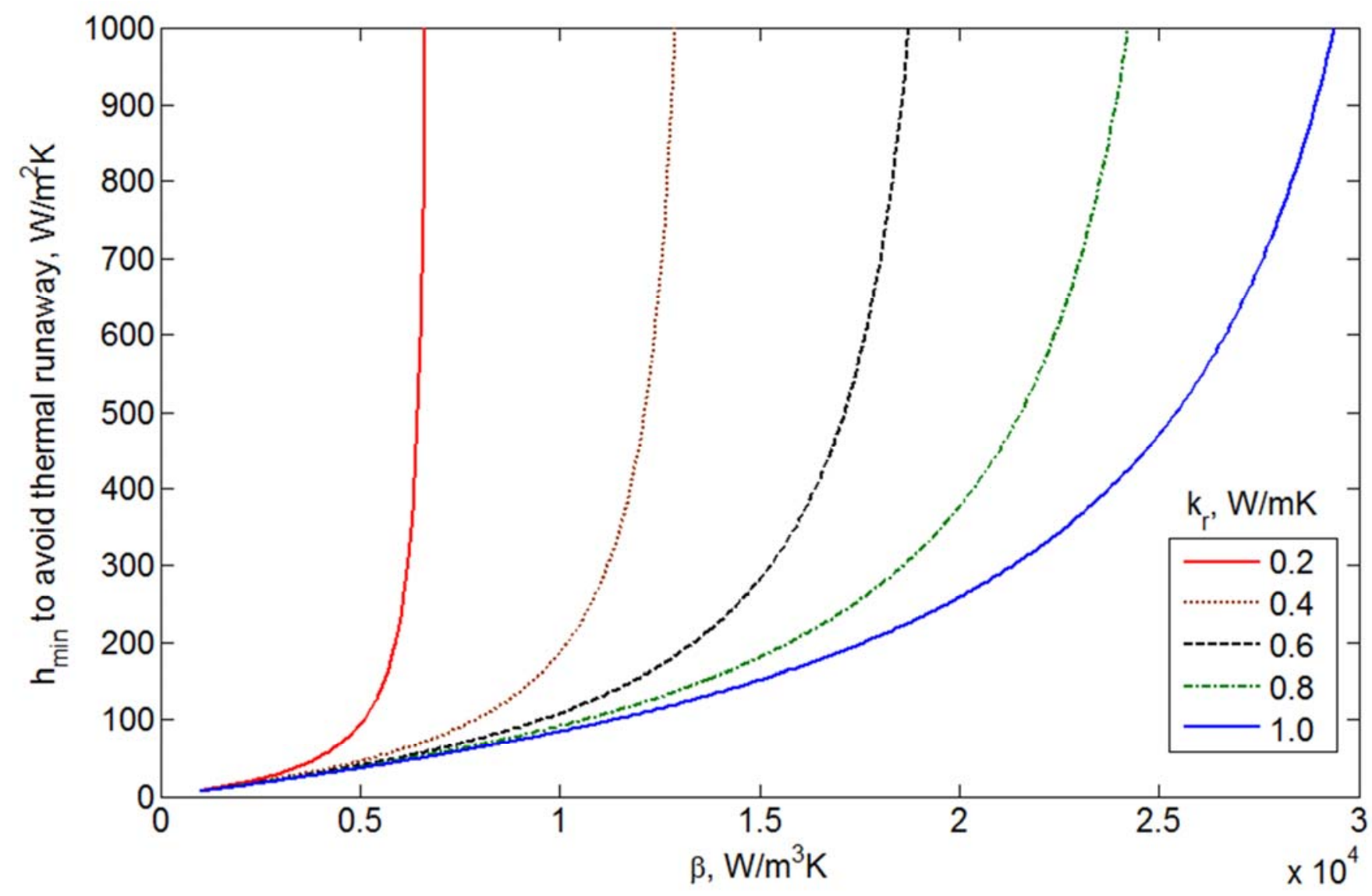

REVISED Figure 7. Minimum heat transfer coefficient needed to prevent thermal runaway as a function of $\beta$ for different values of cell thermal conductivity.

Finally, the two-dimensional thermal runaway model is utilized to predict $\beta_{\max }$, the maximum value of $\beta$ that a cell can sustain as a function of the shape of the cell. Figure 8 plots $\beta_{\max }$ as a function of cell radius for fixed total volume, corresponding to the volume of a 26650 cell. As $R$ increases, $\beta_{\max }$ first reduces, but then starts increasing after reaching a minima. This non-monotonic behavior occurs because for a fixed volume, equation (9) can be rearranged as follows:

$$
\beta_{\text {max }}=\left(\frac{k_{r} \mu_{1}^{2}}{R^{2}}+\frac{\lambda_{1}^{2} \gamma^{2} \pi R^{4}}{V^{2}}\right)
$$

where $V$ is the volume. 
The first term in equation (12) is inversely proportional to $R^{2}$, whereas the second term is directly proportional to $R^{4}$, which explains the non-monotonic behavior in Figure (8). This shows that among all cells of the same volume at given values of $h$ and $k$, the cell of a certain radius sustains the lowest $\beta_{\max }$, and thus has the worst possible thermal runaway behavior at the assumed values of $h$ and $k$. This worst-case radius can be easily obtained by differentiating equation (12). For conditions considered here, as shown in Figure 8, this minima occurs at a radius of around $17 \mathrm{~mm}$, which is quite close to that of the commonly used 26650 cell.

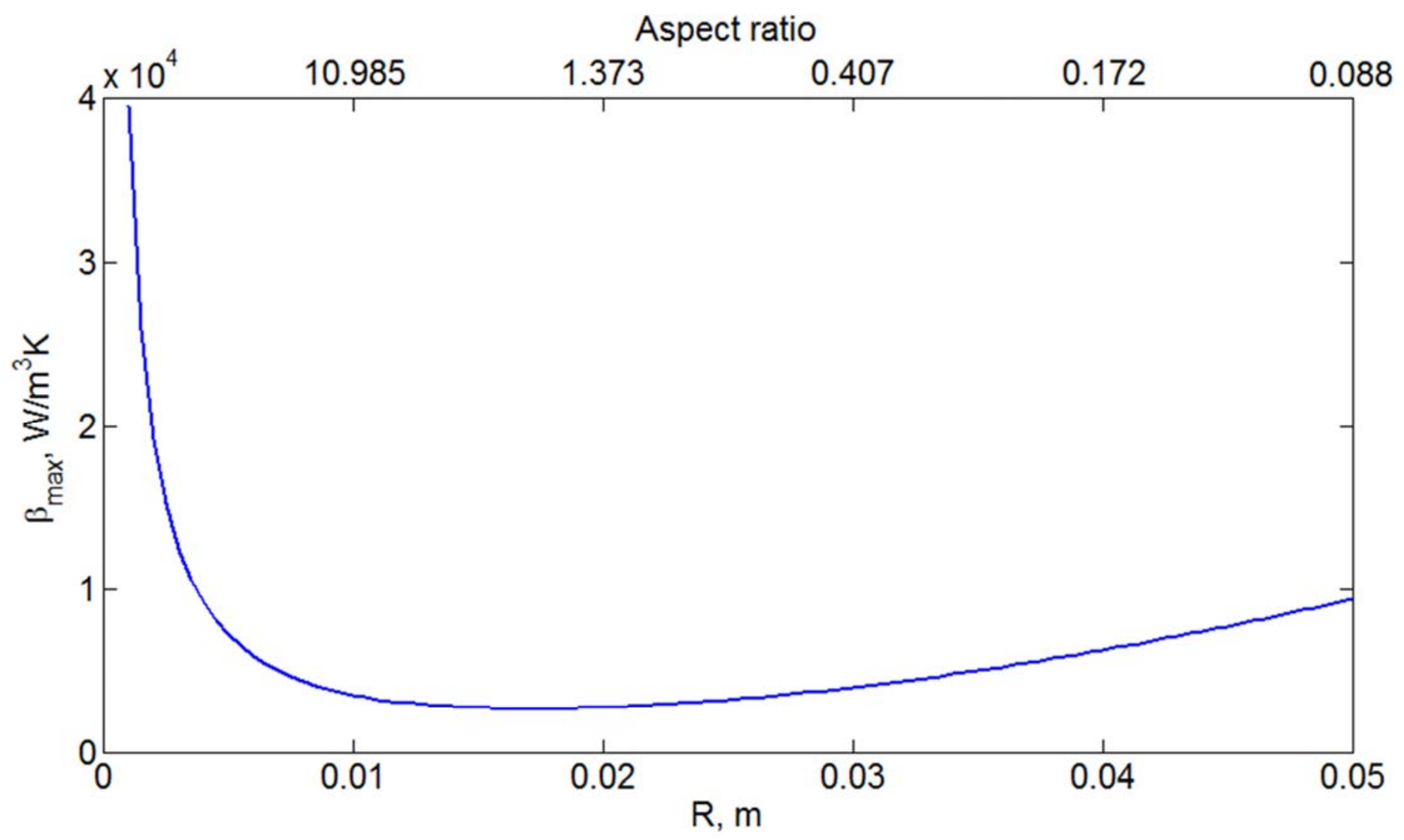

Figure 8. Maximum sustainable $\beta$ as a function of the aspect ratio of a finite-length, cylindrical cell. 


\section{Conclusions}

Thermal runaway is a serious technological challenge that currently limits performance of energy storage and conversion devices. This work experimentally and theoretically demonstrates a new non-dimensional Thermal Runaway Number, $T R N$, that determines whether thermal runaway will occur or not. In addition to improving the fundamental understanding of heat transfer in thermal runaway conditions, this work contributes towards the design of effective thermal management strategies for preventing thermal runaway and for proactively predicting thermal runaway. By quantifying the limits on heat generation rate in given thermal conditions, this work may also contribute towards the design of resilient cell chemistries that better withstand thermal runaway in unfavorable thermal conditions.

\section{ACKNOWLEDGMENTS}

This material is based upon work supported by CAREER Award No. CBET-1554183 from the National Science Foundation.

\section{REFERENCES}

[1] Q. Wang, P. Ping, X. Zhao, G. Chu, J. Sun, C. Chen, J. Power Sources 208 (2012) 210-224.

[2] D. Lisbona, T. Snee, Process Safety \& Env. Protection 89 (2011) 434-442.

[3] R. Spotnitz, J. Franklin, J. Power Sources 113 (2003) 81-100.

[4] P.G. Balakrishnan, R. Ramesh, J. Power Sources 155 (2006) 401-414.

[5] E.P. Roth, D.H. Doughty, J. Power Sources 128 (2004) 308-318.

[6] M.N. Richard, J.R. Dahn, J. Electrochem. Soc. 146 (1999) 2068.

[7] H. Maleki, G. Deng, A. Anani, J. Howard, J. Electrochem. Soc. 146 (1999) 3224-3229.

[8] A. Du Pasquier, F. Disma, T. Bowmer, A.S. Gozdz, G. Amatucci, J.-M. Tarascon, J. Electrochem. Soc. 145 (1998) 472-477. 
[9] D. Aurbach, B. Markovsky, G. Salitra, E. Markevich, Y. Talyossef, M. Koltypin, L. Nazar, B. Ellis, D. Kovacheva, J. Power Sources 165 (2007) pp. 491-499.

[10] P. Biensan, B. Simon, J.P. Peres, A. de Guibert, M. Broussely, J.M. Bodet, F. Perton, J. Power Sources 82 (1999) 906-912.

[11] E.P. Roth, G. Nagasubramanian, 'Thermal stability of electrodes in Li-ion cells,' Technical Report SAND2000-0345J, Sandia National Laboratories, USA, 2000.

[12] Y. Chen, J.W. Evans, J. Electrochem. Soc. 143 (1996) 2708-2712.

[13] Z. Zhang, D. Fouchard, J.R. Rea, J. Power Sources 70 (1998) 16-20.

[14] S. Drake, M. Martin, D.A. Wetz, J.K. Ostanek, S.P. Miller, J.M. Heinzel, A. Jain, J. Power Sources 285 (2015) 266-273.

[15] Y. Ye, L.H. Saw, Y. Shi, K. Somasundaram, A.A.O. Tay, Electrochim. Acta 134 (2014) 327-337.

[16] G.-H. Kim, A. Pesaran, and R. Spotnitz, J. Power Sources 170 (2007) 476-489.

[17] N. Tanaka, W.G. Bessler, Solid State Ionics 262 (2014) 70-73.

[18] M. W. Verbrugge, AIChE J. 41 (1995) 1550-1592.

[19] C.F. Lopez, J.A. Jeevarajan, P.P. Mukherjee, J. Electrochem. Soc. 162 (2015) A2163-2173.

[20] K. Smith, G-H. Kim, E. Darcy, A. Pesaran, Int. J. Energy. Res. 34 (2010) 204-215.

[21] S. Al Hallaj, H. Maleki, J.S. Hong, J.R. Selman, J. Power Sources 83 (1999) 1-8.

[22] K. Shah, S.J. Drake, D.A. Wetz, J.K. Ostanek, S.P. Miller, J.M. Heinzel, A. Jain, J. Power Sources 258 (2014) 374-381.

[23] K. Shah, S.J. Drake, D.A. Wetz, J.K. Ostanek, S.P. Miller, J.M. Heinzel, A. Jain, J. Power Sources 271 (2014) 262-268.

[24] Semenov, N.N., Some Problems in Chemical Kinetics and Reactivity, Princeton University Press, 1959, Vol. 2, Chap. 8.

[25] Q. Wang, J. Sun, G. Chu, Proc. $8^{\text {th }}$ Int. Symp. Fire Safety Sci. (2005) 375-382. 
[26] S. Drake, M. Martin, D.A. Wetz, J.K. Ostanek, S.P. Miller, J.M. Heinzel, A. Jain, J. Power Sources 285 (2015) 266-273.

[27] G. Zhang, L. Cao, S. Ge, C-Y. Wang, C.E. Shaffer, C.D. Rahn, J. Electrochem. Soc., 161 (2014) A1499-1507.

[28] F.P. Incropera, D.P. Dewitt, 'Introduction to heat transfer', 3rd. Ed., Wiley Inc., 2006.

[29] S.J. Drake, D.A. Wetz, J.K. Ostanek, S.P. Miller, J.M. Heinzel, A. Jain, J. Power Sources 252 (2014) 298-304.

[30] J. Zhang, B. Wu, Z. Li, J. Huang, J. Power Sources 259 (2014) 106-116.

[31] M.N. Özisik, Heat Conduction, second ed., John Wiley \& Sons Inc, 1980, ISBN 0-47105481-X.

[32] V. Srinivasan, C.Y. Wang, J. Electrochem. Soc. 150 (2003) A98-A106.

[33] V. Vishwakarma, C. Waghela, Z. Wei, R.S. Prasher, S.G. Nagpure, J. Li, F. Liu, C. Daniel, A. Jain, J. Power Sources 300 (2015) 123-131. 


\section{List of Figures:}

Figure 1. (a) Picture, and (b) Schematic of the experimental setup.

Figure 2. Core temperature of the thermal test cell measured as a function of time for a number of values of $\beta$, ranging from $805 \mathrm{~W} / \mathrm{m}^{3} \mathrm{~K}$ for the left-most (blue) curve to $2685 \mathrm{~W} / \mathrm{m}^{3} \mathrm{~K}$ for the rightmost (red) curve. These data show that thermal runaway occurs when $T R N>1$, and is prevented when $T R N<1$. The value of $T R N$ is shown for each curve. Prediction of temperature profile from the analytical model in Section 2 is also shown for comparison.

Figure 3. Core temperature of the thermal test cell measured as a function of time for fixed $\beta$ and different convective heat transfer conditions.

Figure 4. Experimental measurement of thermal test cell core temperature for (a) two different values of activation energy, $E_{a}$, (b) two different values of convective heat transfer coefficient. Plots also indicate the evolution of TRN with time, and show that thermal runaway occurs when TRN exceeds 1.

Figure 5. Colorplot of TRN in the $\mathrm{h}-\mathrm{k}$ space. The $\mathrm{TRN}=1$ curve that separates safe and unsafe regions is also shown.

Figure 6. (a) $T R N$ as a function of cell thermal conductivity, $k$ for two different values of the convective heat transfer coefficient, $h$; (b) TRN as a function of the convective heat transfer coefficient, $h$ for two different values of the cell thermal conductivity, $k$.

Figure 7. Minimum heat transfer coefficient needed to prevent thermal runaway as a function of $\beta$ for different values of cell thermal conductivity.

Figure 8. Maximum sustainable $\beta$ as a function of the aspect ratio of a finite-length, cylindrical cell. 


\section{List of Supplementary Materials}

1. Special Case for Equation (8): Lumped Thermal Mass

\section{Supplementary Figures:}

Figure S.1: The first root $\mu_{1}$ of equation (8) as a function of the Biot number, $B i$.

Figure S.2: Comparison of experimental measurement of heat generation rate as a function of temperature with the implemented function for two different $\mathrm{Q}(\mathrm{T})$ profiles.

Figure S.3: Comparison of model prediction of temperature rise as a function of time with finiteelement simulation results for two different heating cases. 